\title{
EL VPM: UNA TÉCNICA VARIACIONAL PARA SIMULAR LA RESPUESTA SÍSMICA DE VALLES ALUVIALES SOMEROS
}

\author{
Rafael Avila ${ }^{(1,2)}$, Víctor J. Palencia ${ }^{(3)}$ y Francisco J. Sánchez Sesma ${ }^{(1)}$
}

\begin{abstract}
RESUMEN
Se presenta el Método de Proyección Variacional (VPM, por sus siglas en inglés) para calcular la respuesta sísmica tridimensional de valles aluviales someros. La solución está basada en una formulación variacional de Galerkin de forma débil para el problema de un estrato irregular de geometría suave que sobreyace a un semiespacio elástico. La formulación de Galerkin se aplica mediante una familia de funciones de prueba para establecer la dependencia respecto a la profundidad. Las ecuaciones diferenciales parciales de la elasticidad dinámica se "proyectan" al plano horizontal en superficie donde se resuelven usando un esquema pseudoespectral. Aquí, las ecuaciones son resueltas con diferencias finitas para las derivadas en el tiempo y transformada rápida de Fourier para las derivadas horizontales en el espacio. La irradiación de energía al semiespacio se incluye aproximadamente usando como solución de referencia la respuesta unidimensional en cada sitio. Se dan ejemplos del comportamiento numérico del método y de su exactitud para modelos de valles someros en 2 y 3 dimensiones. Se discute la factibilidad de mejorar el método, particularmente en las condiciones de frontera en la base del modelo.
\end{abstract}

\section{SUMMARY}

The Variational Projection Method (VPM) to compute the seismic response of three dimensional (3D) shallow alluvial valleys is presented. The solution is based on a weak-form variational Galerkin formulation of the problem for a smooth irregular layer overlaying an elastic half-space. The Galerkin method is applied by means of a simple set of trial functions for depth dependence. The partial differential equations of dynamic elasticity in space and time are "projected" into the horizontal free surface plane and are then solved using a pseudospectral scheme: finite differences in time and fast Fourier transform (FFT) to compute spatial horizontal derivatives. By using as reference the 1D solution, the radiation conditions are approximately fulfilled. Numerical examples of the performance and accuracy of the method in 2 and 3D are given. Possible improvements of the method are discussed as well.

Artículo recibido el 2 de octubre de 2001 y aprobado para su publicación el 28 de noviembre de 2002. Se aceptarán comentarios y/o discusiones hasta cinco meses después de su publicación.

(1) Instituto de Ingeniería, UNAM, Ciudad Universitaria, Apdo. 70-472, Coyoacán 04510 México DF., México. rcarrer@imp.mx, sesma@servidor.unam.mx

(2) Instituto Mexicano del Petróleo, Eje Central Lázaro Cárdenas 152, Col. San Bartólo Atepehuacan, Gustavo A. Madero 07730 México D.F., México. rcarrer@imp.mx

(3) División de Matemáticas e Ingeniería, UNAM - Campus Acatlán, Alcanfores y San Juan Totoltepec, Santa Cruz Acatlán, 53150 Naucalpan, Estado de México, México.palencia@servidor.unam.mx 


\section{INTRODUCCIÓN}

Los efectos de sitio pueden producir grandes variaciones espaciales del movimiento sísmico del suelo y ocasionar mayores daños en ciertas zonas. En las últimas tres décadas se han realizado esfuerzos significativos que han ayudado a la caracterización de tales efectos (ver p ej SánchezSesma, 1987; Aki, 1988; Luco et al., 1990). La gran importancia de la amplificación local fue evidente, de nuevo, por el daño producido en varios sismos importantes. Por ejemplo: La ciudad de México en 1985, San Francisco en 1989, y Kobe en 1995. En la actualidad las bases físicas del problema de la amplificación son bien conocidas (ver p ej Singh et al., 1995). Los trabajos más importantes al respecto han estado concentrados en el problema bidimensional. Esto ofreció algunas explicaciones a las observaciones. Sin embargo, con objeto de mejorar los estudios cuantitativos de la respuesta de un sitio, es deseable que en el modelado se tome en cuenta la naturaleza tridimensional del problema. La variabilidad espacial y la polarización del movimiento del suelo observadas en la Ciudad de México han sido interpretadas como efectos 3D (PérezRocha et al.,1991).

Se han propuesto varios métodos para estudiar la propagación de ondas sísmicas en valles aluviales en 3D (ver p ej Sánchez-Sesma y Luzón, 1995). En términos generales el Método de Elementos de Frontera (BEM) permite obtener resultados confiables. En algunas circunstancias un análisis con BEM garantiza una buena descripción de los efectos 3D de configuraciones simplificadas. Este método proporciona muy buenos resultados, útiles para calibrar otros procedimientos. Entre sus limitaciones se encuentra su restricción al tratamiento de medios homogéneos pues, salvo por algunos casos (Sánchez-Sesma et al., 2001), no se cuenta en general con las funciones de Green para medios heterogéneos. Los elementos finitos y las diferencias finitas permiten tratar materiales heterogéneos y pueden manejar virtualmente cualquier configuración geométrica. De hecho las simulaciones más realistas, hasta ahora, son las efectuadas con el método de diferencias finitas. El estudio de Frankel (1993) de la respuesta del valle de San Bernardino en California y las simulaciones de Olsen et al., (1995) para la cuenca de Los Ángeles, ilustran bien este hecho. Las soluciones rigurosas (Horike et al., 1990) y aproximadas (Graves y Clayton, 1992) para estructuras del basamento elásticas y acústicas respectivamente, también ilustran las grandes dificultades en la simulación del problema. Si bien sus resultados son alentadores, las simulaciones exactas pueden ser muy costosas, en términos de tiempo de procesamiento, aun con el empleo de supercomputadoras (ver $\mathrm{p}$ ej Furumura y Takenaka, 1996; Olsen et al., 1997). Por ello creemos que existe un grupo de procedimientos prácticos simplificados que deben tomarse en cuenta para el cálculo de los efectos de sitio en valles aluviales someros.

En este trabajo presentamos al VPM como un método práctico para simular en forma aproximada la respuesta sísmica de valles aluviales someros en 3D. Este método ya ha sido aplicado en el cálculo de la respuesta sísmica tridimensional para algunas zonas del valle de Osaka Japón, durante el sismo de Hyogo-Ken Nanbu (Kobe) del 17 de Enero de 1995 (SánchezSesma et al., 1996) y considerando la respuesta del basamento (Perrot et al., 1995). Nuestra aproximación busca vencer las dificultades de cálculo antes mencionadas. El diseño toma en cuenta una estructura fina como la observada en la respuesta en frecuencia de los modelos 2D en valles aluviales, (Sánchez-Sesma et al., 1993) la cual nos indica que existe un fuerte 
acoplamiento entre la respuesta 1D y las ondas superficiales generadas localmente en este tipo de configuraciones. Con este fin se propuso una aproximación experimental que usa expansiones de modos locales (Ávila et al., 1993). Por otra parte, se ha mostrado que las formulaciones variacionales del tipo Galerkin son eficientes y exactas para los problemas de la propagación de ondas (p ej Faccioli et al., 1996). Una formulación de Galerkin de forma débil es la base de un método riguroso en el dominio de la frecuencia llamado Método de Solución Directa (DSM) (Geller et al., 1990; Geller y Ohminato, 1994; Geller y Hatori, 1995). Con el DSM se han resuelto un grupo de problemas de interés sismológico con gran exactitud.

Con el fin de aprovechar la extensión lateral del problema, usamos una formulación variacional débil y aplicamos localmente el método de Galerkin para "proyectar" el campo de ecuaciones de la elasticidad dinámica dentro de un plano horizontal sobre la superficie del modelo. Usamos también una familia completa de funciones de prueba que cumplen condiciones de ortogonalidad, para establecer la dependencia con la profundidad. En consecuencia, nuestro Método de Proyección Variacional produce un grupo de ecuaciones "proyectadas" en las cuales la dependencia de las coordenadas horizontales y el tiempo se preservan. Estas ecuaciones se resuelven entonces con el método pseudoespectral: las derivadas en el tiempo se aproximan con un esquema centrado de segundo orden, mientras que las derivadas espaciales se calculan usando transformadas de Fourier. Aplicamos este procedimiento para los sedimentos y suponemos una variación suave de la interfaz valle-basamento. El problema puede ser formulado de dos maneras: (1) por especificación del movimiento en la interfaz (usando teoría de rayos y el método de Haskell), lo cual significa que las reflexiones tardías corresponderán al basamento rígido y la energía perdida por radiación hacia el medio exterior puede ser tomada en cuenta sólo aproximadamente con operadores de tiempo; y (2) por especificación de algunos esfuerzos en la interfaz en términos de fronteras parcialmente reflejantes. Estos dos caminos proporcionan resultados alentadores, pero establecer cuál es el mejor es materia de nuestra investigación en la actualidad. La formulación aquí presentada generó cálculos que resultaron ser mucho más rápidos que los procedimientos ya existentes. Damos algunos ejemplos del comportamiento numérico y de la exactitud del VPM para modelos de valles aluviales en 2 y 3D, así como algunas comparaciones con soluciones conocidas, como las del Método Indirecto de Elementos de Frontera (IBEM).

\section{MÉTODO DE PROYECCIÓN VARIACIONAL}

\section{Formulación del problema}

Considere un valle aluvial somero tridimensional sobre la superficie de un semiespacio elástico, como se muestra en la Fig. 1. El material de los sedimentos es elástico y tiene una forma arbitraria, pero suave, descrita por el espesor $h(x, y)$. Se asume que los sedimentos son mucho más blandos que el semiespacio que los subyace. 


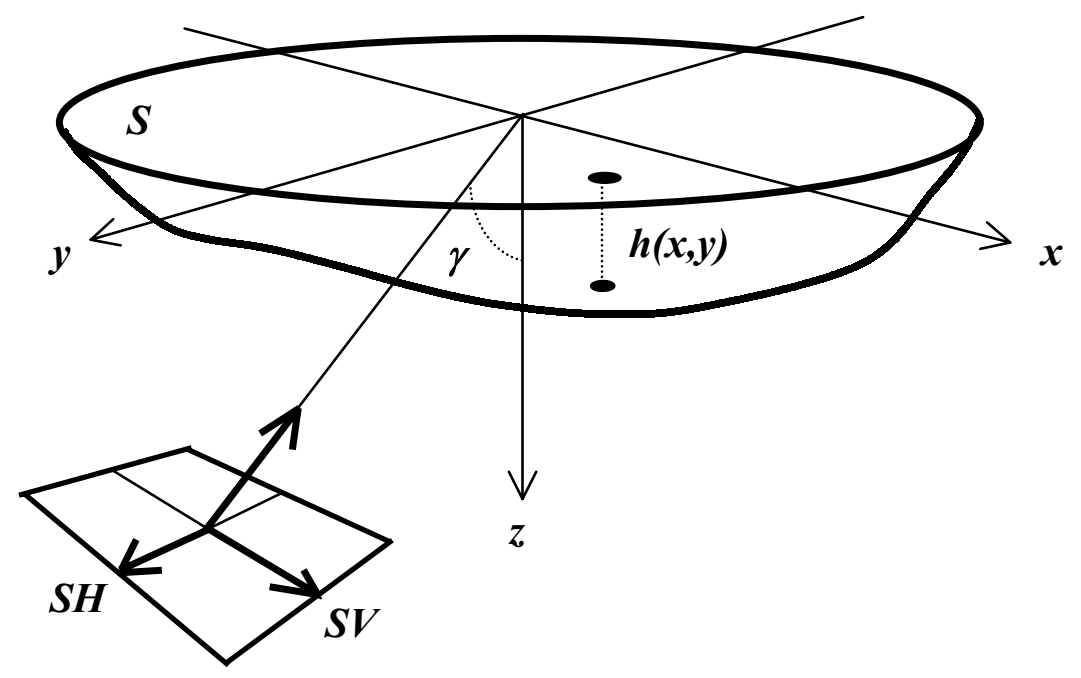

Figura 1. Valle aluvial sometido a la incidencia de una onda plana.

Las ecuaciones de campo en ausencia de fuerzas de cuerpo pueden escribirse, para los sedimentos, por medio de la ley de Newton:

$\frac{\partial \sigma_{i j}}{\partial x_{j}}=\rho \frac{\partial^{2} u_{i}}{\partial t^{2}} ; \quad i=1, \ldots, 3$

donde $\sigma_{i j}$ es el tensor de esfuerzos, $u_{i}$ el vector de desplazamiento, $x_{i}$ las coordenadas cartesianas, $\rho$ la densidad de masa y $t$ el tiempo. En lo que sigue se pueden usar las siguientes equivalencias: $x_{1}=x, x_{2}=y, x_{3}=z$ y $u_{1}=u, u_{2}=v, u_{3}=w$. Adicionalmente, el tensor de esfuerzos está dado por la ley de Hooke:

$\sigma_{i j}=C_{i j k l} \frac{\partial u_{k}}{\partial x_{l}}$

donde $C_{i j k l}$ es el tensor elástico (para materiales isótropos $C_{i j k l}=\lambda \delta_{i j} \delta_{k l}+\mu\left(\delta_{i k} \delta_{j l}+\delta_{i l} \delta_{j k}\right)$ ).

El campo de desplazamiento elástico en los sedimentos se propone con la forma:

$u_{i}(x, y, z, t)=u_{i}^{(0)}(x, y, z, t)+u_{i}^{\prime}(x, y, z, t)$.

donde $u_{i}^{(0)}($.$) es un movimiento de referencia conocido en la interfaz h(x, y)$ y $u_{i}^{\prime}($.$) es el$ movimiento adicional que se busca en el estrato. Sustituyendo la ec. (3) en la ec. (1) se puede escribir 


$$
\frac{\partial \sigma_{i j}}{\partial x_{j}}+f_{i}=\rho \frac{\partial^{2} u_{i}^{\prime}}{\partial t^{2}}
$$

con

$$
f_{i}=\frac{\partial \sigma_{i j}^{(0)}}{\partial x_{j}}-\rho \frac{\partial^{2} u_{i}^{(0)}}{\partial t^{2}}
$$

que es el campo forzante debido al movimiento de referencia, $u_{i}^{(0)}(\cdot)$.

La condición de frontera libre de esfuerzos en la superficie $z=0$ puede aproximarse como,

$$
\sigma_{z j}=\sigma_{z j}^{(0)}+\sigma_{z j}^{\prime}=0
$$

Nótese que $\sigma_{z j}^{(0)}$ depende sólo de $x, y$ y $t$.

El campo de desplazamiento buscado se escribe ahora como

$$
u_{i}^{\prime}(x, y, z, t)=\sum_{n=1}^{N} U_{i}^{n}(x, y, t) \varphi^{n}(z)
$$

donde $U_{i}^{n}(\cdot)$ son funciones de las coordenadas horizontales $(x, y)$ y del tiempo, mientras $\varphi^{n}(z)$ son funciones de prueba dadas de la profundidad. La ec. (7) expresa la solución $u_{i}^{\prime}($.$) en$ términos de la combinación lineal de las funciones de prueba.

\section{Formulación de Galerkin de forma débil}

Considere ahora el operador de Galerkin,

$$
\int_{0}^{h}\left[\frac{\partial \sigma_{i j}^{\prime}}{\partial x_{j}}+f_{i}-\rho \frac{\partial^{2} u_{i}^{\prime}}{\partial t^{2}}\right] \varphi^{m}(z) d z=0
$$

Esta ecuación puede ser vista como la forma fuerte del método de Galerkin para las ecuaciones elásticas a lo largo de una línea vertical con longitud $h(x, y)$. Para expresar la ec. (8) en la forma de un operador débil se integra por partes y se escribe: 
$\int_{0}^{h}\left\{\left[\frac{\partial \sigma_{x i}^{\prime}}{\partial x}+\frac{\partial \sigma_{y i}^{\prime}}{\partial y}+f_{i}-\rho \frac{\partial^{2} u_{i}^{\prime}}{\partial t^{2}}\right] \varphi^{m}(z)-\left(\sigma_{z i}^{\prime}+\sigma_{z i}^{(0)}\right) \frac{\partial \varphi^{m}}{\partial z}\right\} d z+\left[\left(\sigma_{z i}^{\prime}+\sigma_{z i}^{(0)}\right) \varphi^{m}(z)\right]_{0}^{h}=0$

porque $\sigma_{z i}^{(0)}$ no depende de $z$. De acuerdo con la ec. (6) la única contribución del segundo término en la ec. (9) proviene de $z=h$ si $\varphi^{m}(h) \neq 0$.

\section{Las ecuaciones proyectadas}

Si $\varphi^{m}(h)=0$, entonces $u_{i}^{\prime}(\cdot)=0$ y el movimiento de referencia $u_{i}^{(0)}(\cdot)$ puede considerarse como el movimiento real de la interfaz valle-basamento. Si estos movimientos son dados y corresponden a la solución unidimensional en la interfaz, la solución en los primeros tiempos de respuesta concuerda efectivamente con la condición $u_{i}{ }^{\prime}(\cdot)=0$ en $z=h$. Para tiempos de respuesta posteriores la frontera es totalmente reflejante y corresponde a una base rígida.

Entre las diversas familias de funciones completas en el intervalo $(0, h)$ se puede seleccionar la bien conocida familia de cosenos:

$\varphi^{n}(z)=\cos \lambda_{n} z$

con $\lambda_{n}=(2 n+1) \pi / 2 h$. Es claro que $\varphi^{m}(h)=0$. Más aún, estas funciones y sus derivadas (con respecto a $z$ ) forman, respectivamente, sendos conjuntos ortogonales en el intervalo de interés, esto es:

$$
\begin{aligned}
& \int_{0}^{h} \varphi^{m} \varphi^{n} d z=\frac{h}{2} \delta_{m n}, \\
& \int_{0}^{h} \frac{\partial \varphi^{m}}{\partial z} \frac{\partial \varphi^{n}}{\partial z} d z=\lambda_{m}^{2} \frac{h}{2} \delta_{m n}, \\
& \int_{0}^{h} \varphi^{m} d z=\frac{h}{2} \frac{4}{\pi} \frac{(-1)^{m}}{2 m+1}, \\
& \int_{0}^{h} \varphi^{m} \frac{\partial \varphi^{n}}{\partial z} d z=a_{m n},
\end{aligned}
$$

donde $\delta_{m n}$ es la delta de $\operatorname{Kronecker}\left(\delta_{m n}=1\right.$ si $m=n ; \delta_{m n}=0$ si $m \neq n$ ) y 


$$
a_{m n}= \begin{cases}-\frac{1}{2} & \text { si } m=n \\ -\frac{2 n+1}{4}\left[\frac{1+(-1)^{m+n}}{m+n+1}-\frac{1-(-1)^{m-n}}{m-n}\right] & \text { si } m \neq n\end{cases}
$$

Estas propiedades son útiles para simplificar la "proyección" de las ecuaciones de campo de la elasticidad sobre el plano horizontal en la superficie $z=0$.

Introduciendo la ec. (7) en la ec. (9), considerando las ecs. (2) y (3) y tomando en cuenta que, aun cuando $h$ sea función de $x$ y de $y$, se desprecian las derivadas de $h$, se puede escribir:

$$
\begin{gathered}
U_{t t}^{m}-\alpha^{2} U_{x x}^{m}-\beta^{2} U_{y y}^{m}+\beta^{2} \lambda_{m}^{2} U^{m}-\left(\alpha^{2}-\beta^{2}\right) V_{x y}^{m}-\left(\left(\alpha^{2}-2 \beta^{2}\right) a_{m n}-\beta^{2} a_{m n}\right) W_{x}^{n} \frac{2}{h} \\
=\frac{1}{\rho} f_{1} \frac{4}{\pi} \frac{(-1)^{m}}{2 m+1}+\frac{2}{\rho h} \sigma_{z x}^{(0)}, \\
V_{t t}^{m}-\beta^{2} V_{x x}^{m}-\alpha^{2} V_{y y}^{m}+\beta^{2} \lambda_{m}^{2} V_{m}-\left(\alpha^{2}-\beta^{2}\right) U_{x y}^{m}-\left(\left(\alpha^{2}-2 \beta^{2}\right) a_{m n}-\beta^{2} a_{m n}\right) W_{y}^{n} \frac{2}{h} \\
=\frac{1}{\rho} f_{2} \frac{4}{\pi} \frac{(-1)^{m}}{2 m+1}+\frac{2}{\rho h} \sigma_{z y}^{0}, \\
W_{t t}^{m}-\beta^{2} W_{x x}^{m}-\beta^{2} W_{y y}^{m}+\alpha^{2} \lambda_{m}^{2} W^{m}+\left(\left(\alpha^{2}-2 \beta^{2}\right) a_{m n}-\beta^{2} a_{m n}\right)\left[U_{x}^{n}+V_{y}^{n}\right] \frac{2}{h} \\
=\frac{1}{\rho} f_{3} \frac{4}{\pi} \frac{(-1)^{m}}{2 m+1}+\frac{2}{\rho h} \sigma_{z z}^{(0)},
\end{gathered}
$$

que es un grupo de ecuaciones diferenciales parciales acopladas y "proyectadas". Aquí $U_{1}^{n}=U^{n}$, $U_{2}^{n}=V^{n}, U_{3}^{n}=W^{n}$ y los subíndices de $U, V$ y $W$ significan derivadas parciales. Las derivadas espaciales se calculan usando la transformada de Fourier mediante el algoritmo FFT. La matriz $a_{m n}$ resulta de la integral de $\varphi^{n}(z)$ y de su derivada, como se indica en las ecs. (14) y (15).

Puede verificarse que el acoplamiento entre las funciones horizontales en varios órdenes $(n=0,1, \ldots, N)$ surge después de la primera derivada "variacional" con respecto a $z$. Por otra parte, la segunda derivada de, digamos, $U^{m}$ respecto a $z$ está dada por el producto $-\lambda_{m}^{2} U^{m}$.

Con objeto de introducir amortiguamiento, la forma más sencilla consiste en tomar el operador $\partial / \partial t+\gamma$ en lugar de la derivada en el tiempo. Por ejemplo, bajo esta consideración, la segunda derivada $U_{t t}^{n}$ puede leerse como 
$U_{t t}^{n}=\frac{\partial^{2} U^{n}}{\partial t^{2}}+2 \gamma \frac{\partial^{2} U^{n}}{\partial t}+\gamma^{2} U^{n}$

$\mathrm{y}$, discretizando, en el tiempo $j$ tenemos:

$\left(U_{t t}^{j}\right)=\left[\left(1+\gamma \Delta t+\frac{1}{2} \gamma^{2} \Delta t^{2}\right) U^{n j+1}-2 U^{n j}+\left(1-\gamma \Delta t+\frac{1}{2} \gamma^{2} \Delta t^{2}\right) U^{n j-1}\right] \Delta t^{-2}$.

Puede mostrarse que $\gamma \approx \pi f_{p} / Q$ para un factor de calidad $Q$ en la vecindad de la frecuencia $f_{p}$. De la ec. (20) y sus equivalentes para $V_{t t}$ y $W_{t t}$, es posible calcular las funciones $U, V$ y $W$ en los puntos de la malla horizontal en el tiempo $j+1$ en función de sus valores en el tiempo $j-1$ y las derivadas espaciales en el tiempo $j$.

El cálculo de las derivadas horizontales se lleva a cabo con el algoritmo FFT usando todos los puntos de la malla, esto es, en un esquema global. Este enfoque pseudoespectral requiere, por lo menos, entre 2 y 3 puntos en la malla por longitud de onda mínima (las diferencias finitas pueden requerir hasta 8 puntos para obtener la misma exactitud). Finalmente, las ecs. (3) y (7) permiten calcular los desplazamientos en la superficie.

\section{Condiciones de frontera absorbente.}

Si se escoge un conjunto de funciones de prueba tales que $\varphi^{m}(h) \neq 0$ es posible introducir condiciones de frontera absorbente aproximadas en términos de una sustitución apropiada del esfuerzo en profundidad. La familia de cosenos:

$\varphi^{n}(z)=\cos \frac{n \pi z}{h}$

cumple con las condiciones de ortogonalidad y es completa en $(0, h)$. En $z=h$ su valor es $(-1)^{n}$.

La solución es relativamente sencilla en el dominio de la frecuencia. Supóngase un medio estratificado como el de la Fig. 2, sometido a un campo incidente de ondas planas $S H$.

El campo está dado por:

$v^{(i)}=v_{0}(\omega) e^{-i k x} e^{+i \eta_{E} z} e^{+i \omega t}$,

donde, 


$$
k=\frac{\omega}{\beta_{E}} \operatorname{sen} \gamma \quad \eta_{E}=\frac{\omega}{\beta_{E}} \cos \gamma,
$$

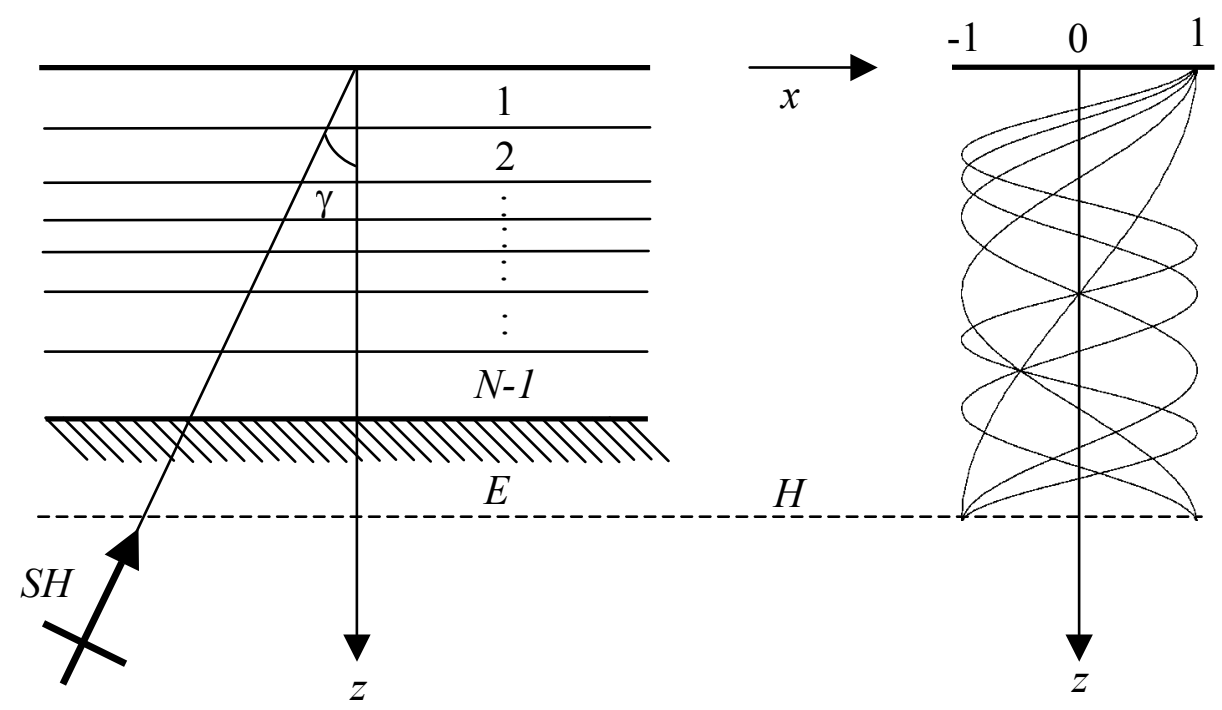

Figura 2. Medio estratificado sometido a la incidencia de un campo de onda plana $S H$ (izq.). Familia de funciones de prueba utilizadas para fronteras absorbentes (der.).

siendo $k$ el número de onda horizontal y $\eta_{E}$ el número de onda vertical; $\omega$ es la frecuencia angular y $\beta_{E}$ la velocidad de fase de las ondas $S H$ en el semiespacio $E$. Si se define al desplazamiento como un movimiento de referencia conocido, $V^{(i)}$, debido solamente al campo incidente, más un desplazamiento, $V(z)$, dado, en función de la profundidad, se puede escribir:

$v=\left(V^{(i)}+V(z)\right) e^{-i k z} e^{+i \omega t}$

es decir, $V^{(i)}=v_{0}(\omega) e^{+i \eta_{E} z}$ y $V(z)$ como el desplazamiento que será calculado utilizando una formulación de Galerkin de forma débil, en la cual se incluye a las funciones de prueba de la ec. (21). De esta manera, $V(z)$ se escribe como:

$V(z)=\sum_{n=0} A_{n} \phi^{n}(z)$

Recordando la ecuación en el dominio de la frecuencia, se tiene que:

$\frac{\partial}{\partial x}\left(\mu \frac{\partial v}{\partial x}\right)+\frac{\partial}{\partial z}\left(\mu \frac{\partial v}{\partial z}\right)+\rho \omega^{2} v=0$

y, adoptando un esquema de Galerkin de forma fuerte, sustituyendo la ec. (24) en la ec. (26) y operando las derivadas queda: 


$$
\int_{0}^{h}\left[-k^{2} \mu\left(V^{(i)}+V\right)+\frac{\partial}{\partial z}\left(\mu \frac{\partial V^{(i)}(z)}{\partial z}+\mu \frac{\partial V}{\partial z}\right)+\rho \omega^{2}\left(V^{(i)}+V\right)\right] \varphi^{m}(z) d z=0,
$$

la cual es una integral sólo en la dirección vertical, que se resuelve por partes e incluye los valores de los coeficientes $A_{n}$ de la ec. (25), los cuales son las incógnitas del sistema dado por:

$$
\begin{gathered}
\int_{0}^{h}\left[-k^{2} \mu \sum A_{n} \varphi^{n} \varphi^{m}-\mu \sum A_{n} \varphi_{z}^{n} \varphi_{z}^{m}+\rho \omega^{2} \sum A_{n} \varphi^{n} \varphi^{m}\right] d z+\left(\mu \frac{\partial V}{\partial z}\right)_{h} \varphi^{m}(h) \\
=\int_{0}^{h}+k^{2} \mu V^{(i)} \varphi^{m} d z+\mu \frac{\partial V^{(i)}}{\partial z}(h) \varphi^{m}(h)+\int_{0}^{h} \mu \frac{\partial V^{(i)}}{\partial z} \varphi_{z}^{m} d z-\int_{0}^{h} \rho \omega^{2} V^{(i)} \varphi^{m} d z
\end{gathered}
$$

que es un sistema cerrado en forma débil en el cual las derivadas parciales de $V$ en $z=h$ son

$$
\left(\mu \frac{\partial V}{\partial z}\right)_{h}=-i \omega \rho_{E} \beta_{E} V_{h} .
$$

La ec. (28) representa un sistema de $m$ por $n$ ecuaciones que se resuelve numéricamente. Cabe resaltar que esta ecuación contiene la información detallada de cada estrato en cuestión y que la responsabilidad de la reconstrucción de las ondas reflejadas y transmitidas se deja únicamente a las funciones de prueba. Así se tiene una representación aproximada de los coeficientes de reflexión y transmisión en cada intervalo del medio estratificado y, a su vez, una buena aproximación de las fronteras absorbentes en la base del modelo.

Un ejemplo de la aplicación de estas fronteras se da en la Fig. 3, donde se muestra la función de transferencia del desplazamiento para un modelo de 3 estratos con las mismas propiedades (prueba de transparencia), ante incidencia vertical de ondas $\mathrm{SH}$. La grafica ilustra el método propuesto con línea discontinua y con línea continua la solución exacta.

Como se puede observar la aproximación es buena. Esto corrobora que la base de funciones es la correcta y que con un orden no mayor a $n=20$ se puede obtener una buena representación de los desplazamientos en el tiempo, además de que posiblemente pueden suprimir la condición de base rígida del VPM. Los resultados mostrados en este artículo corresponden a la formulación original (base rígida), que utiliza la familia de funciones de la ec. (10). La formulación correspondiente a la familia de funciones de la ec. (21) está siendo extendida a casos bidimensionales para estudiar los efectos de dispersión producidos por grietas o cavidades (Iturrarán-Viveros, comunicación personal). 

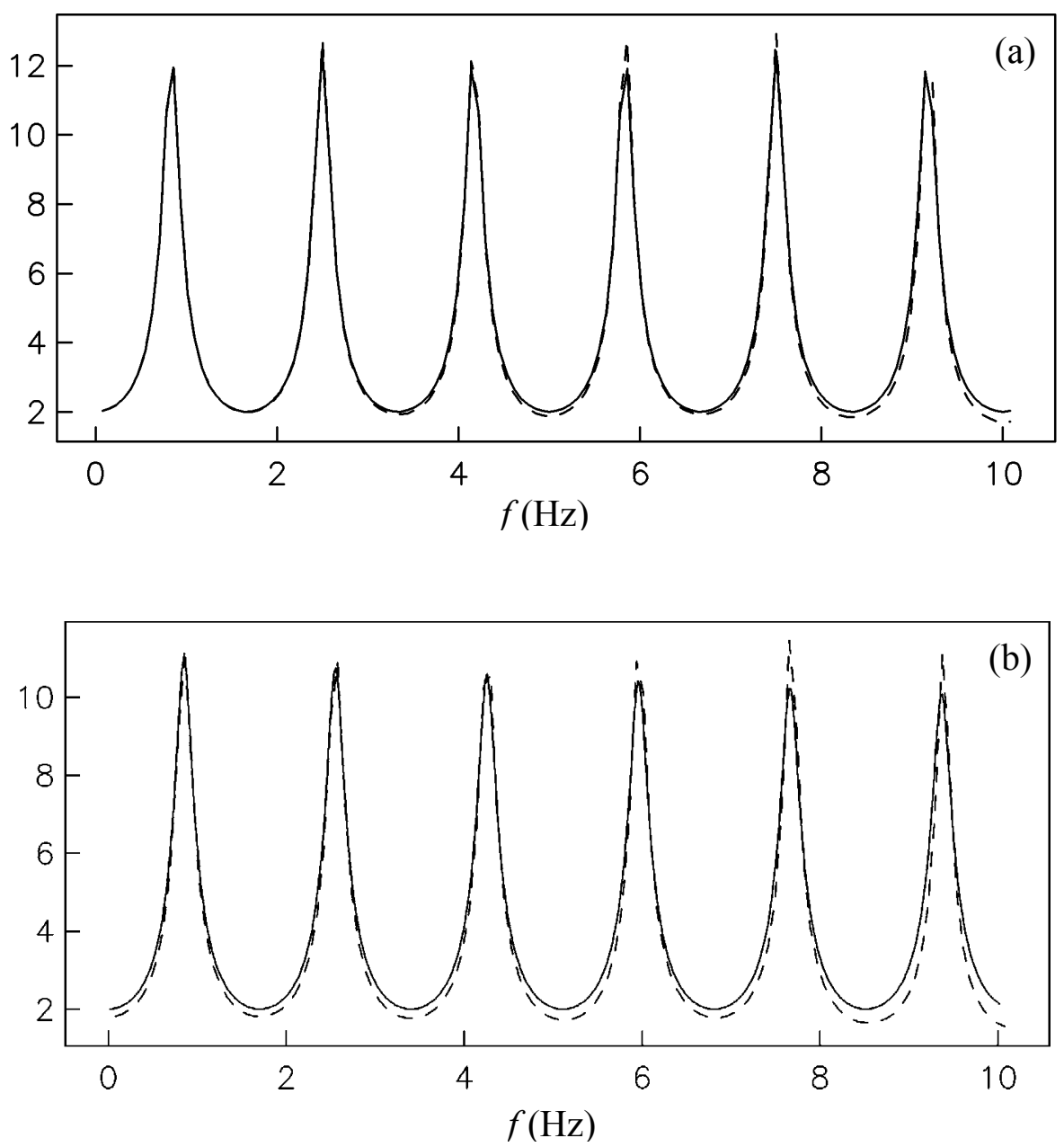

Figura 3. Función de Transferencia del desplazamiento para 3 estratos. $\rho / \rho_{E}=0.4, \beta / \beta_{E}=0.4$ y $h=300 \mathrm{~m}$. (a) Incidencia normal de ondas $S H\left(\gamma=0^{\circ}\right)$. (b) Incidencia oblicua $(\gamma=30)$

\section{RESULTADOS NUMÉRICOS}

Se realizaron algunas pruebas y comparaciones en el caso bidimensional, para verificar el comportamiento numérico, los tiempos de cálculo y la exactitud del método propuesto. Se tomaron como referencia otras técnicas de solución como las correspondientes a las funciones de Green y al Método Indirecto de Elementos de Frontera (IBEM).

La Fig. 4 muestra el modelo de un valle en forma trapezoidal propuesto por Kawase y Aki (1989) que se tomó para comparar al VPM contra un método que utiliza funciones de Green. La Fig. 5 muestra los sismogramas sintéticos obtenidos para el modelo del valle citado. 


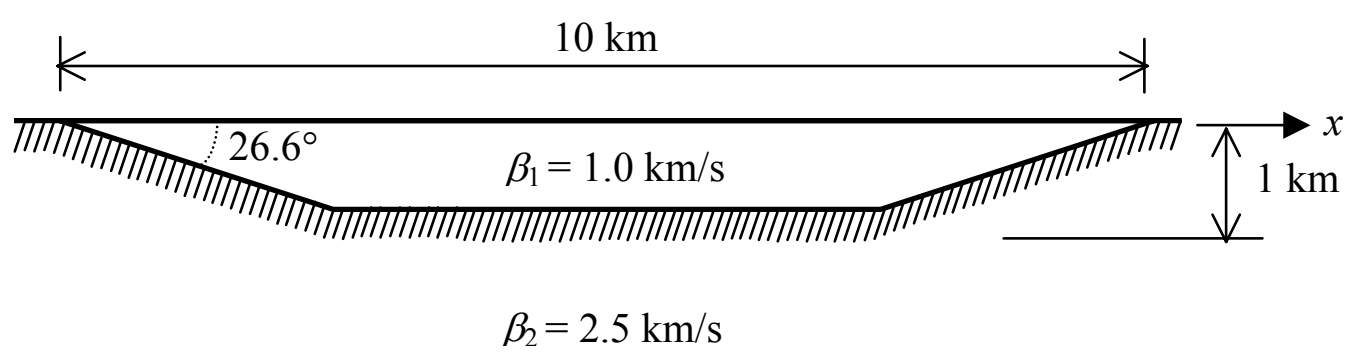

Figura 4. Modelo de valle trapezoidal

Esta figura muestra las ondas generadas por las esquinas del depósito propagándose horizontalmente, las cuales corresponden al modo fundamental de las ondas de Love como lo señalaron Kawase y Aki (1989). La amplitud de las ondas de Love es menor que la de la onda directa. Sin embargo, estas ondas superficiales hacen que la duración total de los registros se vea prolongada. Los intervalos de tiempo entre el arribo de la onda directa y la onda de Love son menores de 10 segundos cerca de las esquinas. Los sismogramas corresponden a una incidencia vertical de ondas $S H$ y un pulso de Ricker con $f_{c}=0.25 \mathrm{~Hz}(4 \mathrm{~s})$. Es notable la similitud de ambos sintéticos.

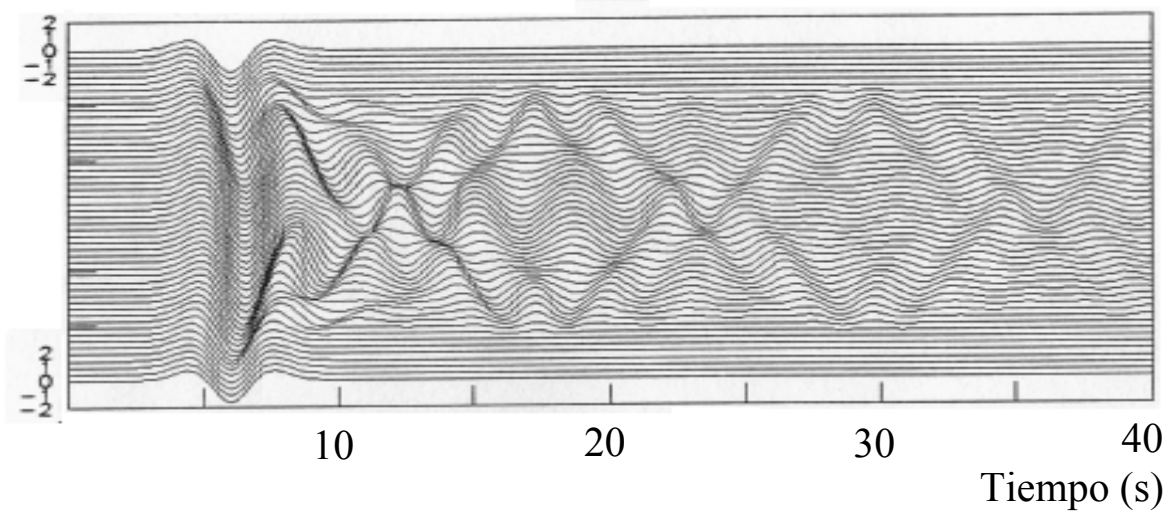

(a)

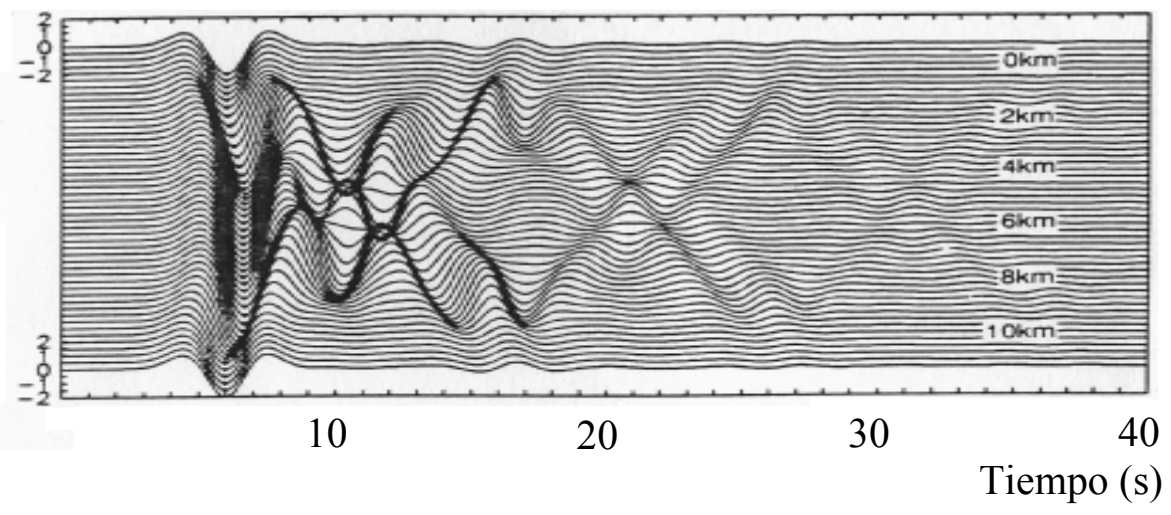

(b)

Figura 5. (a) Sismograma sintético a lo largo de la superficie del depósito de la Fig. 4. (b) El mismo caso que (a), obtenido con el método de funciones de Green utilizado por Kawase y Aki (1989). 
La Fig. 6 muestra la respuesta para un pulso de Ricker con un frecuencia central $f_{c}=0.5 \mathrm{~Hz}$. Las condiciones son las mismas que en la Fig. 5. La amplitud máxima de la onda de Love decrece porque la energía se dispersa entre el modo fundamental y el primer modo superior, como lo demuestran Kawase y Aki (1989).

Si se observa con detenimiento la Fig. 6 podemos resaltar que los sismogramas sintéticos obtenidos con el VPM ofrecen una buena similitud en la respuesta del depósito con respecto del método de referencia. Sin embargo, aunque las trazas representan correctamente la propagación de las ondas dentro del valle, estas muestran un rizamiento a lo largo de toda la respuesta. Esto se debe a que la frecuencia del pulso de Ricker es más alta y nos acercamos a los límites de estabilidad del método (condición de Courant). Por otra parte, la formulación rígida del VPM no permite la correcta absorción de la energía dentro del valle.

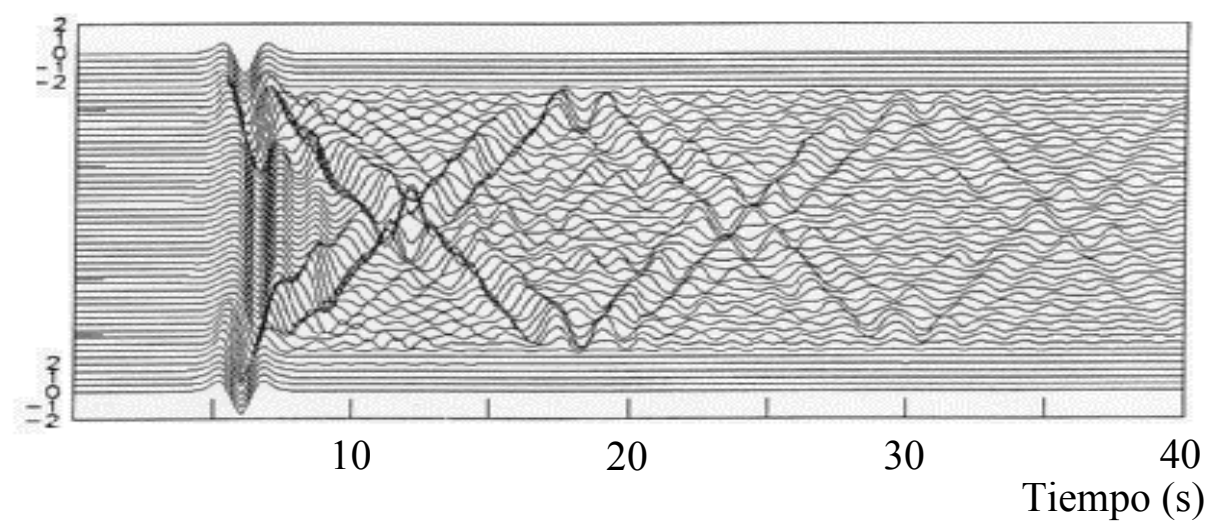

(a)

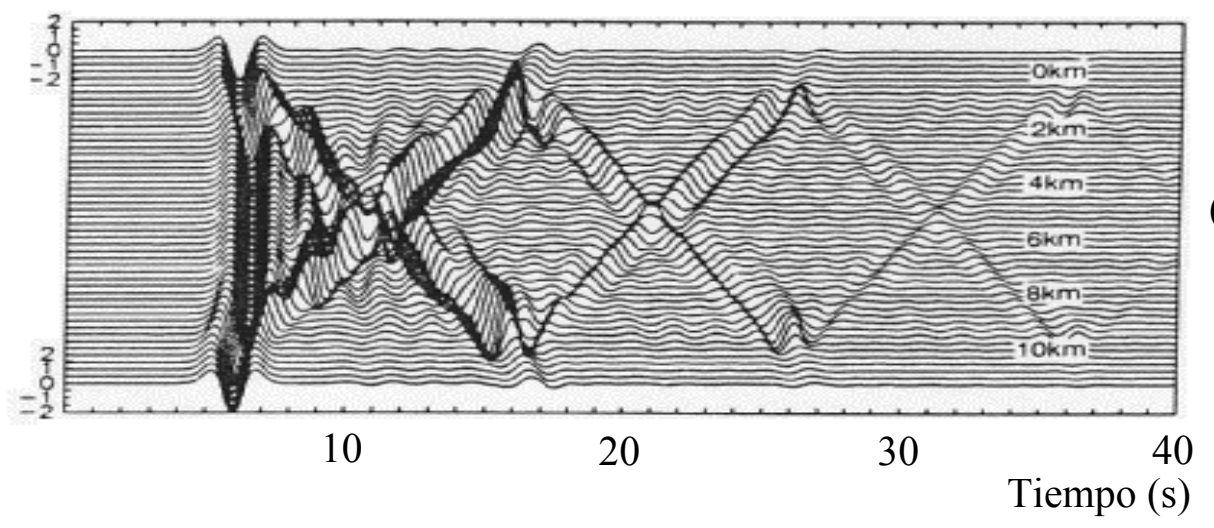

(b)

Figura 6. (a) Sismograma sintético a lo largo de la superficie del depósito de la Fig. 4. (b) El mismo caso que (a), obtenido con un método de funciones de Green.

Enseguida se considera el caso de incidencia de una onda plana $S V$. La Fig. 7 muestra la respuesta del depósito de la Fig. 4 para una onda de este tipo que incide verticalmente con $f_{c}=0.25 \mathrm{~Hz}$. Es muy clara la aparición de trenes sucesivos de ondas en la zona de transición (componente horizontal) y en la parte plana (componente vertical). La diferencia entre la incidencia de ondas $S H$ y $S V$ se debe principalmente al hecho de que las velocidades de fase y de grupo del modo fundamental de la onda de Rayleigh son considerablemente mayores que las de 
las ondas de Love para la frecuencia correspondiente. La onda de Rayleigh generada en las esquinas necesitó solamente 5 segundos para alcanzar la esquina opuesta.

(a)

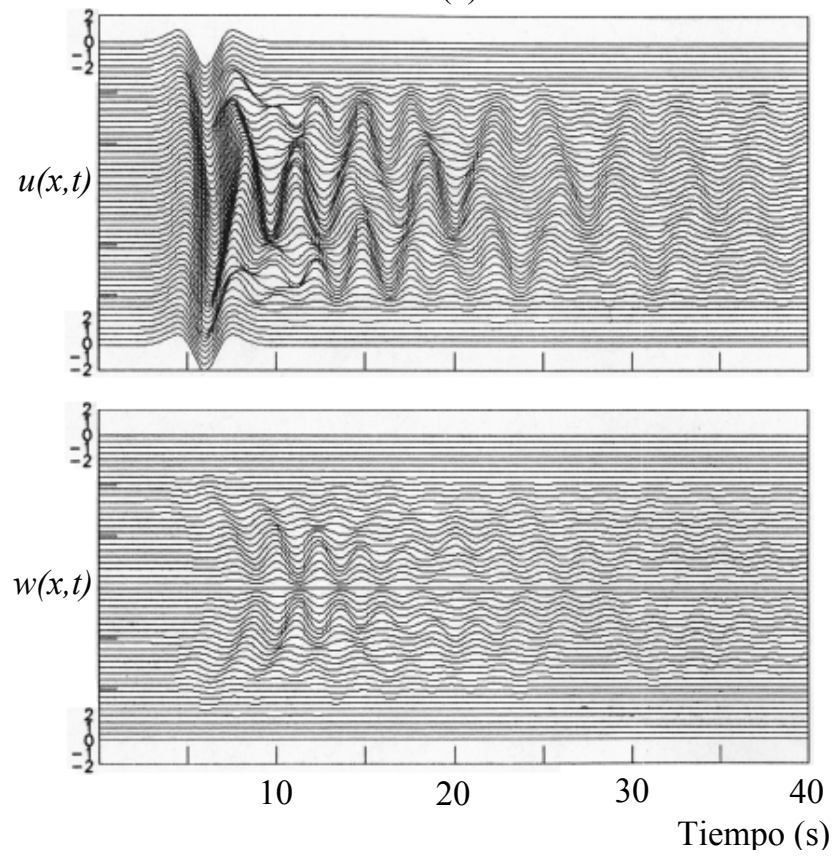

(b)
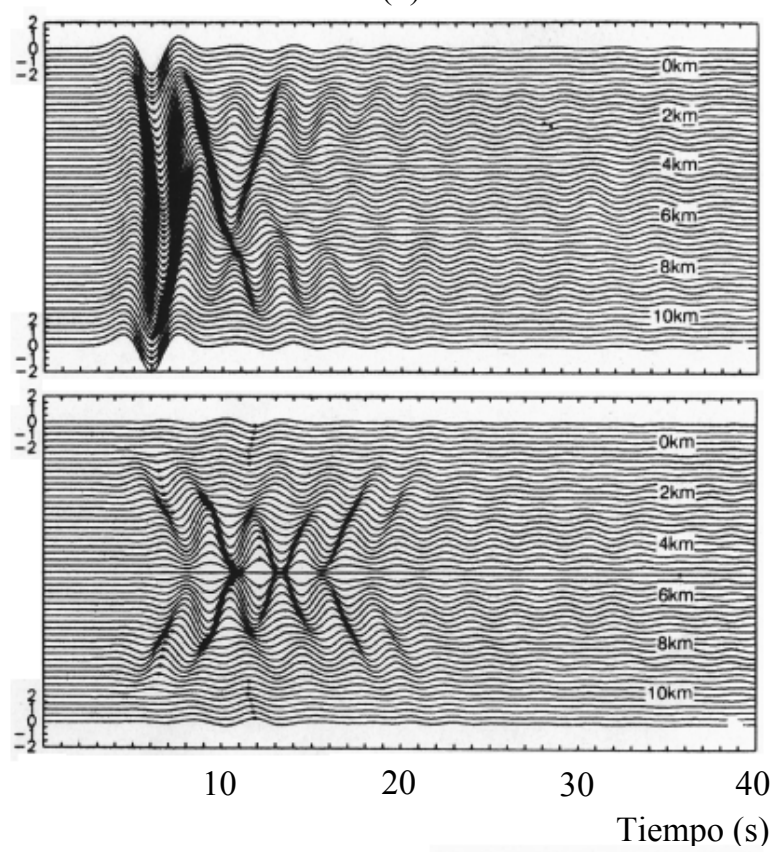

Figura 7. Historias de tiempo de las componentes horizontal $u$, y vertical $w$. (a) Método propuesto; (b) Método de funciones de Green.

Si se analiza la Fig. 8 se puede ver que ante la incidencia de una onda con un contenido de frecuencia más alto ( $f_{c}=0.5 \mathrm{~Hz}$ ) existen ondas de Rayleigh claramente definidas. En este caso el VPM logra reproducir el modo fundamental y el primer modo superior, aunque se nota un tercer frente de ondas. Éste no corresponde a los resultados observados por el método de funciones de Green. De lo anterior resulta claro que aunque el VPM ofrece una velocidad de proceso muy alta, los resultados obtenidos presentan sólo buenas aproximaciones de la realidad. Así, para el mejoramiento de esta técnica es necesario depurar las condiciones de frontera, principalmente en la base del modelo.

También se realizaron algunas comparaciones para el caso tridimensional de un modelo como el de la Fig. 9, que consiste en un valle aluvial somero de forma irregular, ante la incidencia de ondas $S H$. Las velocidades de la onda $S$ en los sedimentos y el semiespacio son $\beta_{R}=1 \mathrm{~km} / \mathrm{s}$ y $\beta_{E}=2.5 \mathrm{~km} / \mathrm{s}$ respectivamente, los coeficientes de Poisson son $v_{R}=0.35$ y $v_{E}=0.25$ y las densidades están relacionadas por $\rho_{R}=0.8 \rho_{E}$, donde $\rho_{E}$ es la densidad del basamento. El factor de calidad en el basamento es de $Q=100$. Este modelo fue propuesto por Sánchez-Sesma y Luzón (1995) quienes enfatizan la buena resolución y calidad de los resultados. Por ello se eligió como un buen modelo de comparación. 

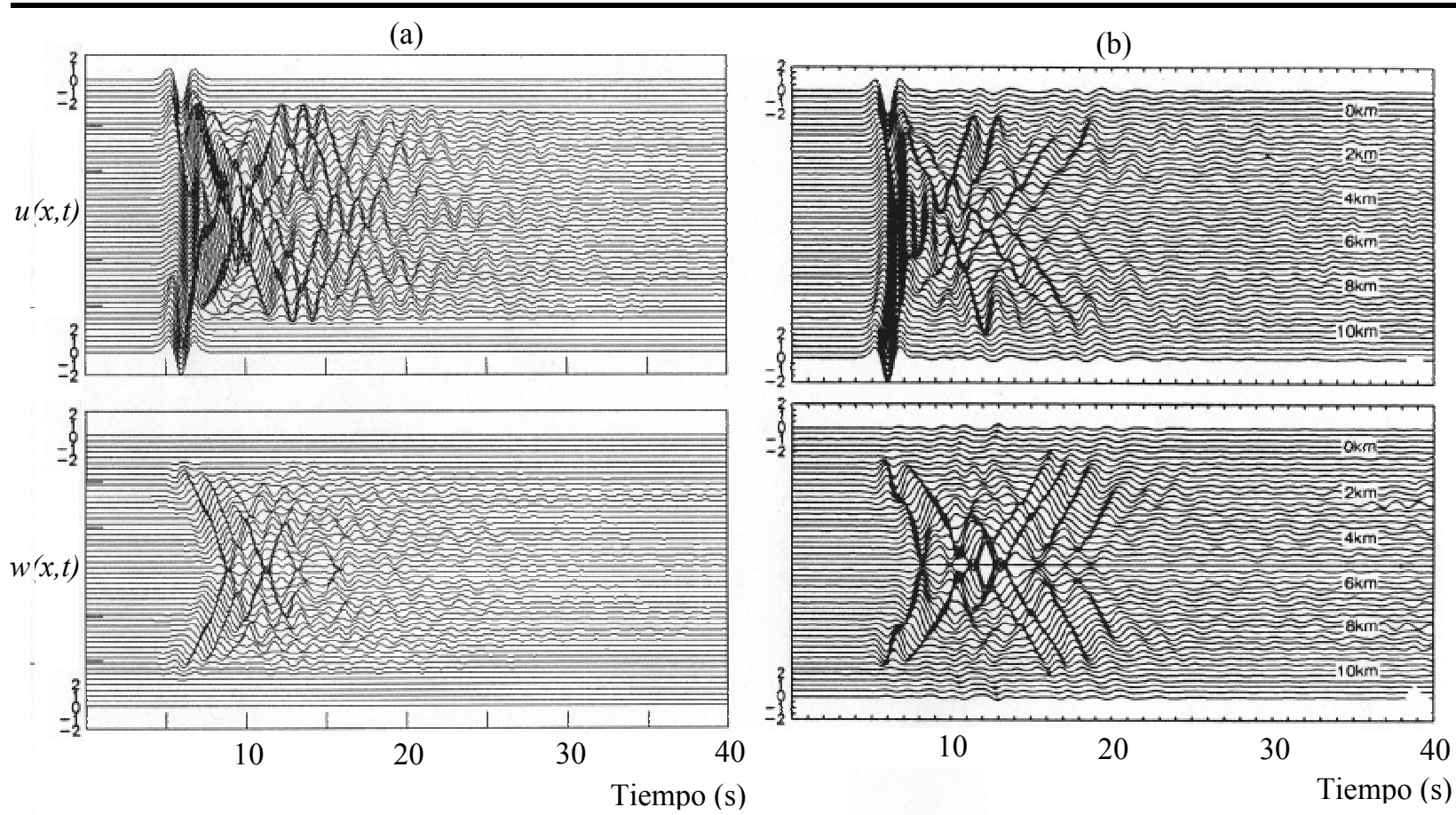

Figura 8. Respuestas $u$ y $w$ para el valle de la Fig. 4. Incidencia vertical de ondas $S V$ con un pulso de Ricker $f_{c}=0.5 \mathrm{~Hz}(2 \mathrm{~s})$. (a) VPM; (b) Funciones de Green.

La Fig. 10 contiene los sismogramas sintéticos obtenidos para un arreglo de detectores ubicados a lo largo de los ejes $x$ e $y$ mostrando las componentes del movimiento en la dirección de $v$ y las componentes horizontal $u$, y vertical $w$, a lo largo del eje $y$. Se trata de una incidencia oblicua de ondas $S H$ con un ángulo de incidencia $\gamma=30^{\circ}$. Se utilizó un pulso de Ricker con un período $t_{p}=3 \mathrm{~s}$. Aquí se presentan los resultados obtenidos con el método propuesto y con el IBEM. En general se observa una buena similitud entre los dos. Los resultados son muy alentadores ya que el VPM representa correctamente la onda incidente y su respuesta unidimensional al entrar en contacto con el valle. Sin embargo, la reproducción de las ondas superficiales de Love resulta más compleja debido a la concentración de energía por la geometría del valle y la formulación rígida del VPM. Por otra parte, el VPM necesitó sólo algunos minutos para su cálculo en una estación de trabajo mientras que el IBEM requirió de 5 días de CPU en la misma estación. Esto hace al VPM un método poderoso, más aún por su formulación directa en el tiempo y su buena resolución 3D, la cual se ilustra a continuación en la Fig. 11.

La Fig. 11 muestra las simulaciones dinámicas para cuatro tiempos de visualización $\left(U_{1}=\right.$ $6, U_{2}=7, U_{3}=8$ y $U_{4}=9 \mathrm{~s}$ ) en las componentes $u, v$ y $w$ del desplazamiento, para una onda $\mathrm{SH}$ que incide con un ángulo de incidencia $\gamma=30^{\circ}$. Se puede observar que el movimiento refleja mayores amplitudes alrededor de los $8 \mathrm{~s}$ para los tres casos mostrados. Estas instantáneas en el tiempo permiten observar los efectos locales que afectan a la propagación de ondas dentro del medio de interés. Así, se pueden obtener resultados confiables de la respuesta sísmica del terreno 


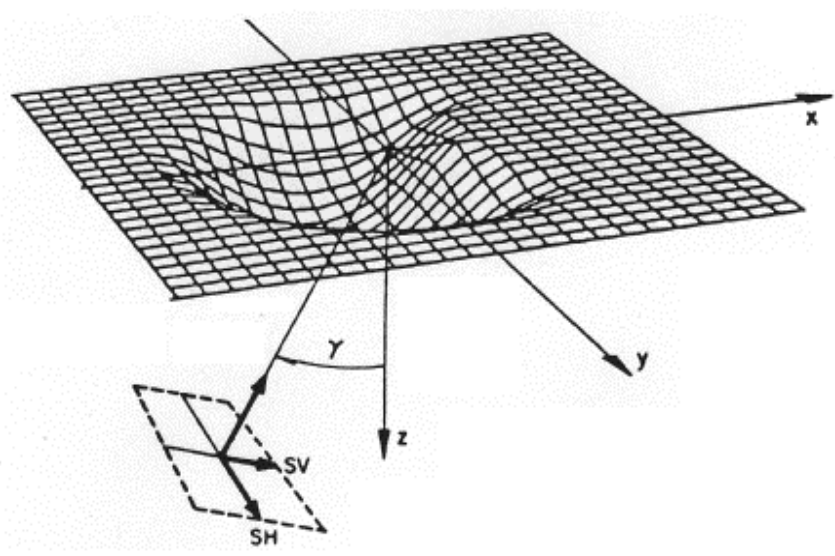

Figura 9. Modelo de valle aluvial somero propuesto por Sánchez-Sesma y Luzón (1995).

para un sitio específico. Por otra parte, habrá que resaltar su aplicación para analizar efectos azimutales en la propagación y generación de ondas superficiales en un valle de nuestro interés, como el de la Ciudad de México.

El objetivo de mostrar las simulaciones dinámicas para el movimiento es que esto permite realizar un análisis tridimensional directamente en el tiempo. La irradiación de energía hacia el semiespacio, la propagación, amortiguamiento y dispersión de las ondas dentro y fuera del valle, constituyen características de suma importancia para la comprensión del fenómeno sísmico. Si se establece una comparación con los análisis de frecuencias de otros autores, se podría entender este fenómeno en mejores condiciones.

Los casos de incidencia $S H$ tratados por medio del VPM muestran una buena correlación con la emisión tardía de pulsos difractados mostrados por el IBEM. Sánchez-Sesma y Luzón (1995) resaltan esta emisión típica de energía difractada, no obstante que un valle con estas características presenta una atenuación geométrica muy fuerte.

Se ha observado que la rapidez de cálculo del VPM sobrepasa por mucho a la de otros métodos y que su aproximación es aceptable para la mayoría de los casos mostrados. Por ello se espera aplicar con éxito el VPM en el futuro, en casos de valles más complejos preferentemente correspondientes a casos realistas como podría ser, eventualmente, el Valle de México. Con esto se pretende aprovechar las ventajas de formulación y facilidad de operación del VPM ante el modelado sísmico de un problema real.

Sin embargo, la investigación en este campo es aún incipiente. Se requiere, mediante el uso de los correspondientes operadores de interfaz, revisar las aproximaciones que pueden proporcionar diversas familias de funciones de prueba y adecuar el problema en el dominio del tiempo para el caso de fronteras absorbentes o dinámicas. Los resultados deberán verificarse contra casos reales. 

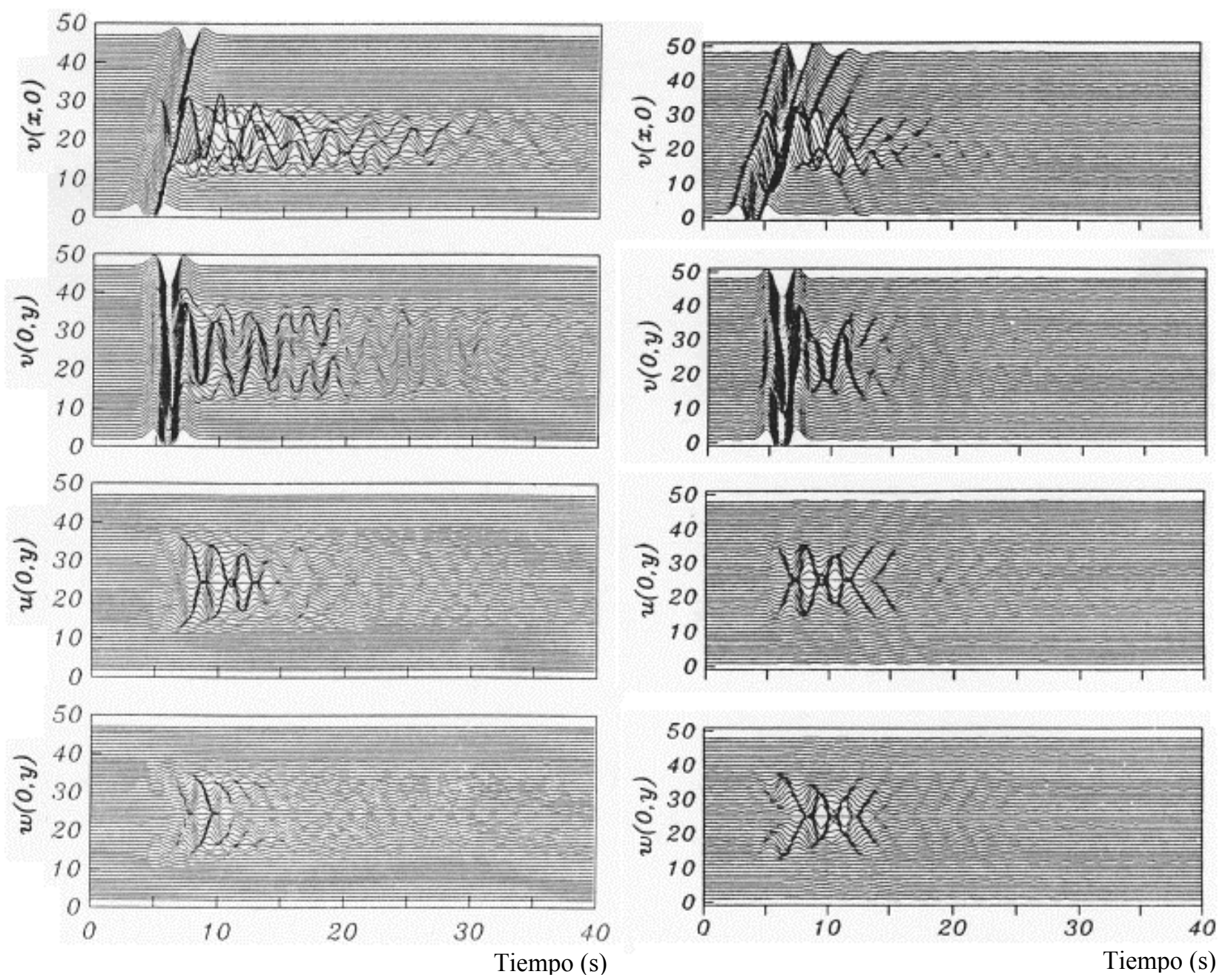

Figura 10. Sismogramas sintéticos para el modelo de la Fig. 9 obtenidos con: (a) VPM y (b) el método indirecto de elementos de frontera (IBEM).

\section{CONCLUSIONES}

Se ha presentado el Método de Proyección Variacional (VPM). Éste calcula la respuesta sísmica tridimensional de valles aluviales someros. El problema se resuelve mediante una aproximación basada en una formulación variacional de Galerkin de forma débil. Se supone un depósito con geometría irregular, de forma suave, donde se aplica el método de Galerkin por medio de un grupo de funciones de prueba para establecer la dependencia con la profundidad. Así se obtiene un grupo de ecuaciones diferenciales que se acoplan y se "proyectan" en el plano horizontal sobre la superficie del modelo donde se resuelven usando un esquema pseudoespectral. Esta aproximación se aplicó a dos modelos de valles aluviales en 2D y 3D, respectivamente. Se utilizó el modelo propuesto por Kawase y Aki (1989), quienes resuelven el problema para el caso bidimensional utilizando un grupo de funciones de Green para explicar las largas duraciones de 


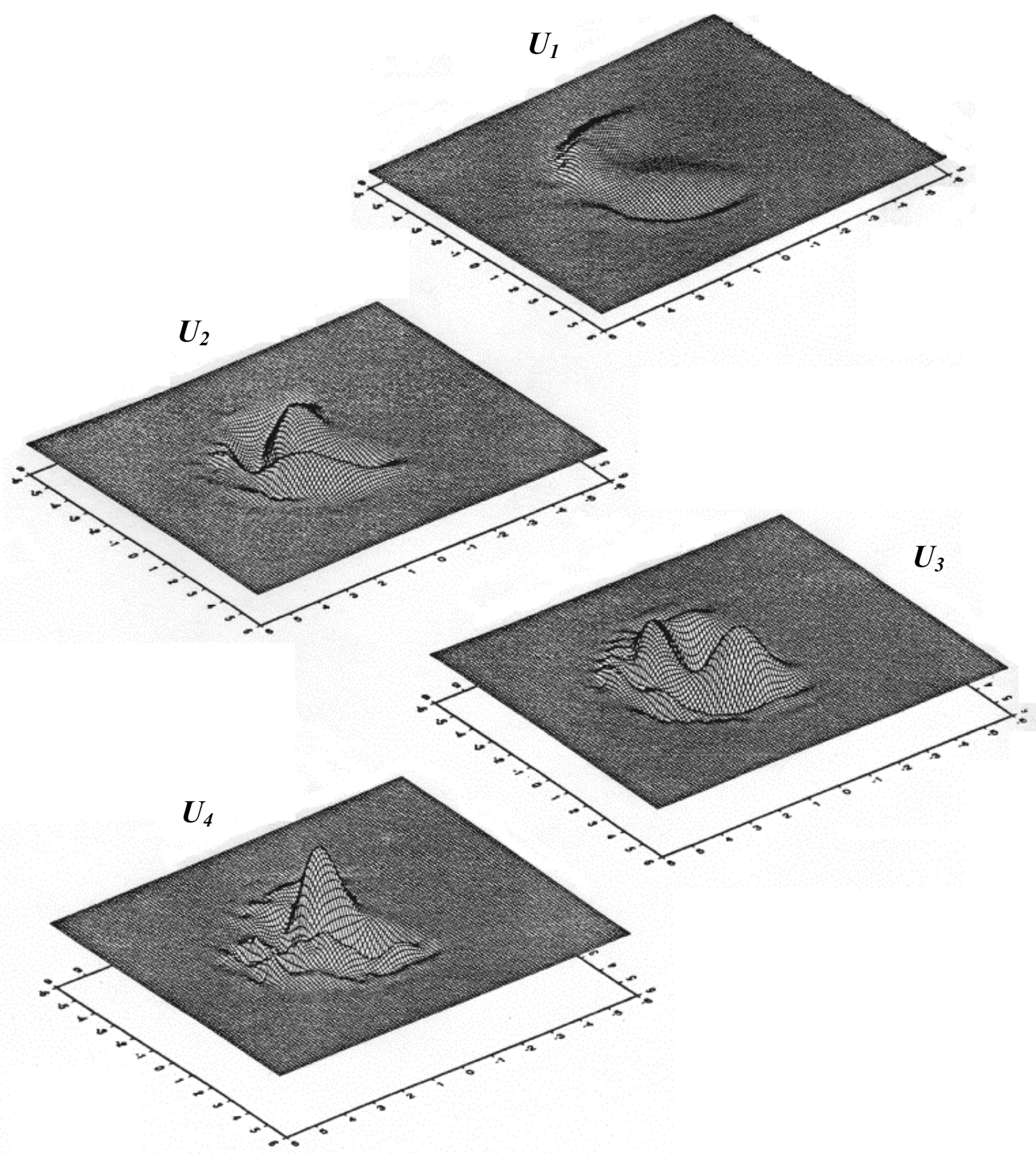

Figura 11. (a) Simulación dinámica, en la componente $u$, para el modelo de la Fig. 9 en cuatro tiempos de visualización. 


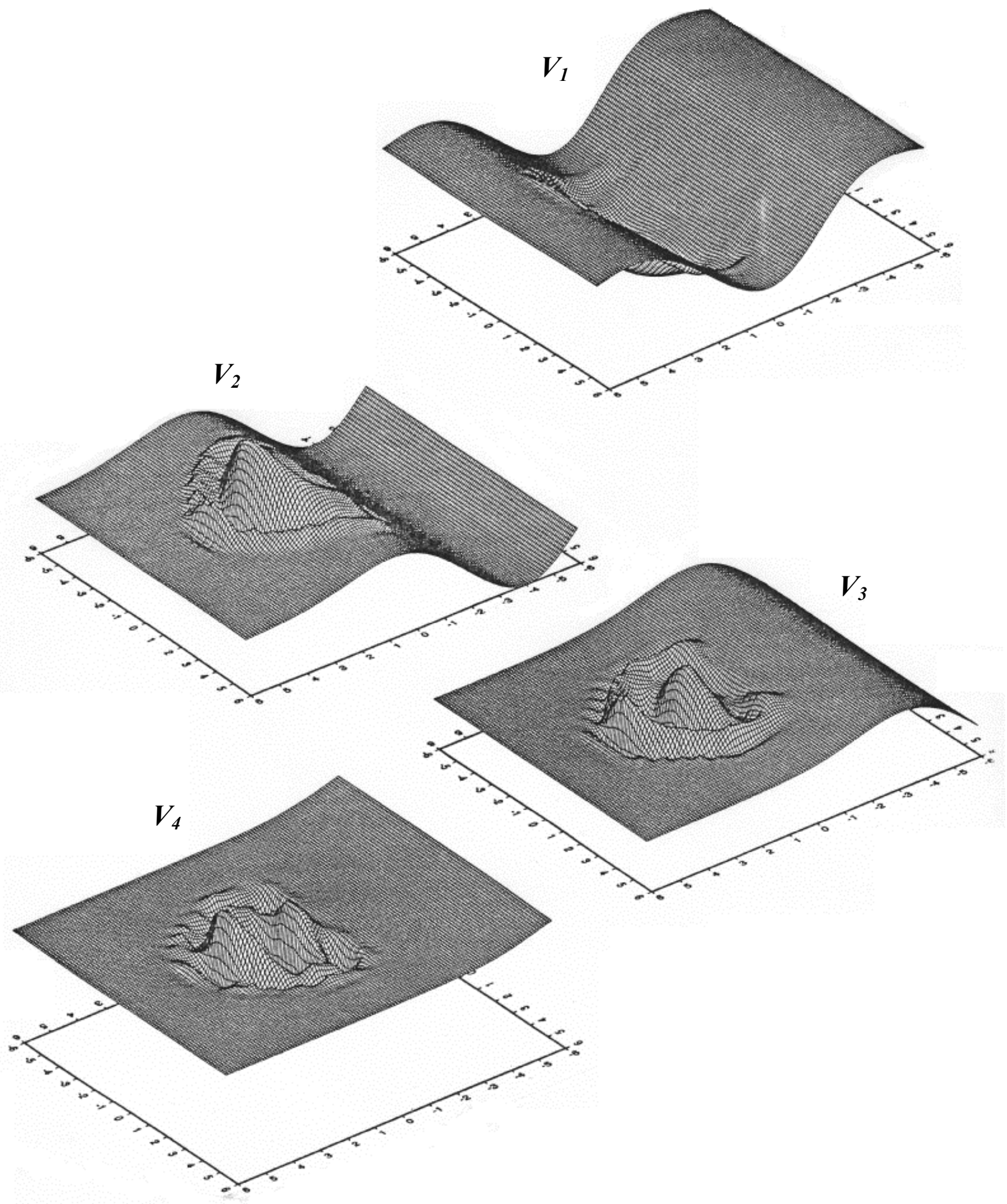

Fig. 11 (b) Componente $v$. 


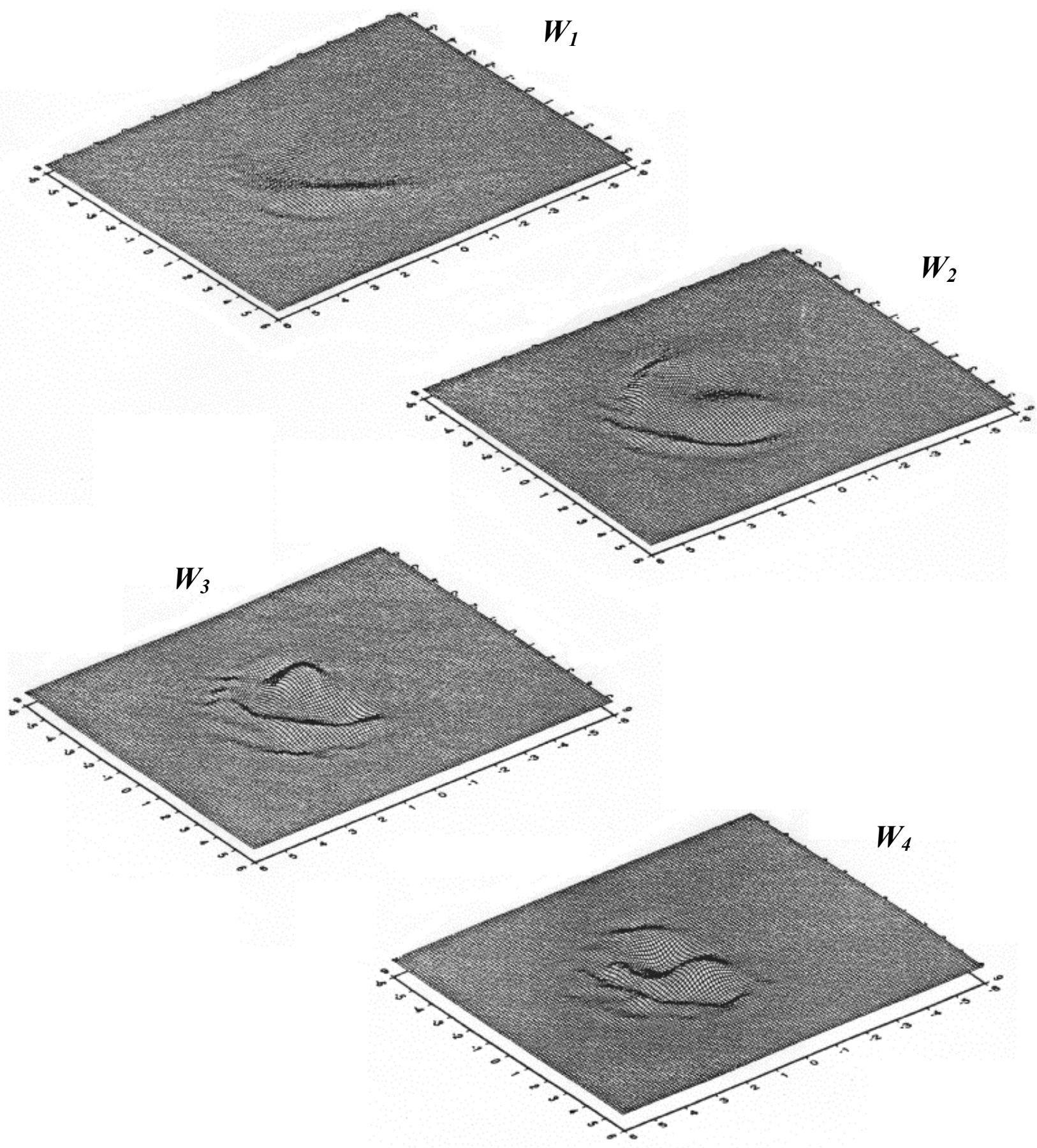

Figura 11. (c) Componente $w$.

los registros de la Ciudad de México. En este caso el acuerdo entre los resultados obtenidos por ambos métodos fue satisfactorio. Así mismo se realizaron algunos cálculos tomando el modelo 
tridimensional propuesto por Sánchez-Sesma y Luzón (1995) para observar la aproximación de resultados entre el método propuesto y el IBEM. El VPM mostró rapidez en los cálculos y buena aproximación en los resultados. El VPM ofrece una buena alternativa para la simulación del movimiento del terreno y constituye una herramienta que puede emplearse para obtener una aproximación práctica y cuantitativa del estudio de la respuesta sísmica de un sitio. El método se encuentra aún en desarrollo y restan algunos puntos importantes que deben resolverse antes de poder aplicarlo para la simulación de casos más realistas. Particularmente, se deben optimar las condiciones de frontera absorbente y así mejorar la solución en el dominio del tiempo.

\section{AGRADECIMIENTOS}

Agradecemos a G. Quiroga-Goode, R. Martin, y K. Irikura por la lectura crítica de este manuscrito y sus comentarios. A J. Bielak, E. Faccioli y R. Geller, por sus sugerencias (sus trabajos proporcionaron ideas para la formulación del método aquí presentado). Parte de los cálculos fue hecha en la CRAY-YMP de la UNAM, México. Este trabajo estuvo auspiciado en parte por DGAPA-UNAM., México, (proy. IN-104998), la Japanese Society for the Promotion of Science, CONACYT, México; por Cray Research Inc., y el Instituto Mexicano del Petróleo.

\section{REFERENCIAS}

Aki, K (1988), "Surface motion of a layered medium having an irregular interface due to incident plane $S H$ waves”, Journal Geophysical Research, Vol. 75, pp. 933-954.

Ávila, R, M Suárez y F J Sánchez-Sesma (1993), "Simulación de la propagación de ondas sísmicas en configuraciones irregulares con un método pseudo-espectral", Memorias del $X$ Congreso Nacional de Ingeniería Sísmica, Puerto Vallarta, Jal., México, pp. 182-189.

Faccioli, E, F Maggio, A Quarteroni y A Tagliani (1996), "Spectral domain decomposition methods for the solution of acoustic and elastic wave equations", Geophysics, Vol. 61, No. 4, pp.1160-1174.

Frankel, A (1993), "Three-dimensional simulations of ground motion in the San Bernardino Valley, California, for hypothetical earthquakes on the San Andreas fault", Bulletin of Seismological Society of America,. Vol. 83, 1020-1041.

Furumura, K y H Takenaka (1996), "2.5 modelling of elastic waves using the pseudospectral method”, Geophysical Journal International, Vol. 124, pp. 820-832.

Geller, R J, T Hara y S Tsuboi (1990), “On the equivalence of two methods for computing partial derivatives of seismic waveforms”, Geophysical Journal International, Vol. 102, pp. 499-502.

Geller, R J y T Ohminato (1994), "Computation of synthetic seismograms and their partial derivatives for heterogeneous media with arbitrary natural boundary conditions using the Direct Solution Method", Geophysical Journal International, Vol. 116, pp. 421-446.

Geller, R J y T Hatori (1995), "DSM synthetic seismograms using analytical trial functions: plane-layered, isotropic, case”, Geophysical Journal International, Vol. 120, pp. 163-172. 
Graves, R W y R Clayton (1992), "Modeling path effects in three dimensional basin structures", Bulletin of Seismological Society of America, Vol. 82, pp. 81-103.

Horike, C (1990), “A finite element method for solving Helmholtz type equations in wave guides and other unbounded domains", Mathematics of Computation, Vol. 39, pp. 209-324.

Kawase, H y K Aki (1989), "A study on the response of a soft basin for incident $S, P$ and Rayleigh waves with special reference to the long duration observed in Mexico City", Bulletin of Seismological Society of America, Vol. 79, pp. 1361-1382.

Luco, J E, H I Wong y F C P De Barros (1990), “Three-dimensional response of a cylindrica1 canyon in a layered half-space", Earthquake Engineering and Structural Dynamics, Vol. 19, pp. 799-817.

Olsen, K B, R J Archuleta y J R Matarese (1995), "Three-dimensional simulation of a magnitude 7.75 earthquake on the San Andreas fault”, Science, Vol. 270, pp. 1628-1632.

Olsen, K B, R Madariaga y R J Archuleta (1997), "Three-dimensional dynamic simulation of the 1992 Landers Earthquake”, Science, Vol. 278, pp. 834-838.

Pérez-Rocha, L E, F J Sánchez-Sesma y E Reinoso (1991), "Three-dimensional site effects in Mexico City: evidences from accelerometric network observations and theoretical results", Memorias, 4th International Conference on Seismic Zonation, Stanford, California, agosto 25-29, Vol. II, pp. 327-334.

Perrot, J, F J Sánchez-Sesma, K Irikura, J L Rodriguez-Zúñiga y R Ávila (1995), “Simulation of seismic response of Osaka basin during the January 17, 1995 Kobe earthquake using the Variational Projection Method", Memorias, AGU Fall meeting, San Francisco.

Sánchez-Sesma, F J (1987), "Site effects on strong ground motion", Earthquake Engineering and Structural Dynamics, Vol. 6, pp. 124-132.

Sánchez-Sesma, F J, R Madariaga y K Irikura (2001), “An approximate elastic 2D Green's function for a constant-gradient medium", Geophysical Journal International, Vol. 146, pp.237248.

Sánchez-Sesma, F J, J Ramos y M Campillo (1993), “An indirect boundary element method applied to simulate the seismic response of alluvial valleys for incident $P, S$ and Rayleigh waves", Earthquake Engineering and Structural Dynamics, Vol. 22, pp. 279-295.

Sánchez-Sesma, F J y F Luzón (1995), "Seismic response of three-dimensional alluvial valleys for incident $P, S$, and Rayleigh waves", Bulletin of Seismological Society of America, Vol. 85, pp. 269-284.

Sánchez-Sesma, F J, K Irikura, J Perrot, J L Rodríguez-Zúñiga y R Ávila (1996), “The Variational Projection Method: A new technique to simulate the seismic response of shallow alluvial valleys", Memorias, $11^{\text {th }}$ World Conference on Earthquake Engineering, Acapulco, México, Artículo No.946, CD-ROM.

Singh, S K, R Quaas, M Ordaz, F Mooser, D Almora, M Torres y R Vázquez (1995), "Is there a truly "hard" rock site in the Valley of Mexico?", Geophysical Research Letters, Vol. 22, pp. 481484. 